\title{
The operator spectrum of the six-dimensional $(1,0)$ theory
}

\section{Eric G. Gimon and Costin Popescu}

California Institute of Technology, Pasadena, CA 91125

E-mail: 'egimon@theory.caitech.edu', popescu@theory.caitech.edui

\begin{abstract}
We study the large $\mathrm{N}$ operator spectrum of the $(1,0)$ superconformal chiral six-dimensional theory with $E_{8}$ global symmetry. This spectrum is dual to the Kaluza-Klein spectrum of supergravity on $A d S_{7} \times S^{4} / \mathbb{Z}_{2}$ with a ten-dimensional $E_{8}$ theory at its singular locus. We identify those operators in short multiplets of $\operatorname{OSp}(6,2 \mid 2)$, whose dimensions are exact for any N. We also discuss more general issues concerning $A d S / C F T$ duality on orbifold supergravity backgrounds.
\end{abstract}

KEYwords: 'I/N Expansion, p-branes, Conformal Field Models in String Theory, Supersymmetry and Duality! 


\section{Contents}

i]. Introduction

2. Compactification geometry

3. Oscillator construction of $\operatorname{OSp}(6,2 \mid 2)$ short multiplets

4. Kaluza-Klein reduction of bulk eleven-dimensional supergravity

5. Kaluza-Klein reduction of $E_{8}$ twisted sector modes

6. Discussion

\section{Introduction}

In the last year important new methods for studying superconformal theories have been developed based on a conjecture by Maldacena [i] $\left[\begin{array}{l}1 \\ 1\end{array}\right]$. This conjecture relates D-dimensional superconformal theories to string theory/M-theory to $A d S_{D+1} \times M$, where $M$ is a compact manifold. Using a prescription given by Gubser, Klebanov and Polyakov [2] and Witten [3] one can associate operators on the D-dimensional boundary with fields in the bulk of $A d S_{(D+1)}$ and compute correlation functions for these operators using supergravity.

In the spirit of much of the modern work in string duality, our goal in this paper is not the presentation of further evidence for the AdS/CFT correspondence, but rather to use this correspondence to determine the unknown spectrum of operators for interacting superconformal theories. Of particular interest for us is the

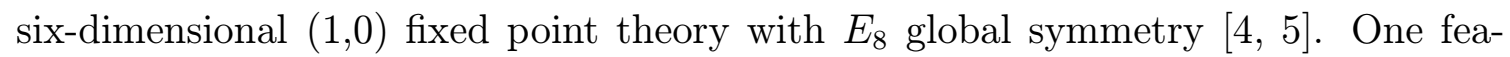
ture which makes this theory particularly interesting is that it has both a Coulomb branch along which states charged under $E_{8}$ decouple, and a Higgs branch with broken $E_{8}$ global symmetry. The transition from one branch to the other can be used to connect four-dimensional theories with different numbers of chiral fields [i, $[\overline{6}]$. Unfortunately this otherwise useful field theory has no known lagrangean description 
(see [iiㅖ). There does exist a discrete light-cone quantized description for the $(1,0)$

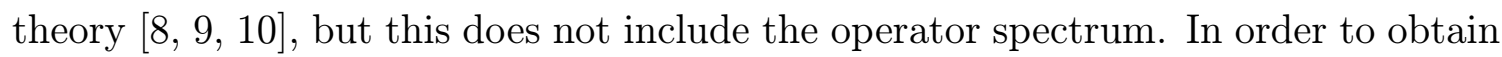
better control over the properties of the $(1,0)$ superconformal fixed point we use $A d S / C F T$ duality to extract its spectrum of chiral operators.

To derive the spectrum of chiral operators, we determine the spectrum of KaluzaKlein modes for the M-theory background in the large $\mathrm{N}$ limit where supergravity is valid. ${ }^{1}$ The $\mathrm{K}-\mathrm{K}$ modes couple to gauge invariant operators on the AdS boundary with dimensions which are either protected or receive $1 / \mathrm{N}$ corrections, and provide a description of the chiral operator spectrum of the $(1,0)$ theory which remains useful for small N. Other six-dimensional $(1,0)$ theories (with different global symmetries)

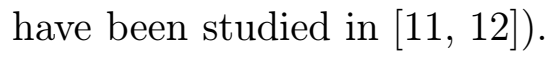

Finding the supergravity background appropriate for describing the $(1,0)$ theory involves orbifolding the dual background for the interacting large $\mathrm{N}(2,0)$ six-

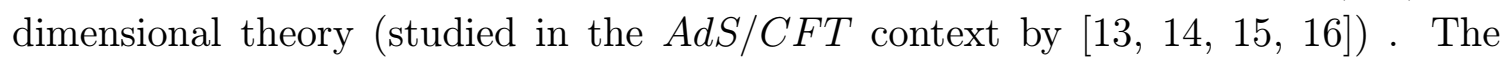
orbifold process brings interesting new aspects to the AdS/CFT story (see for ex-

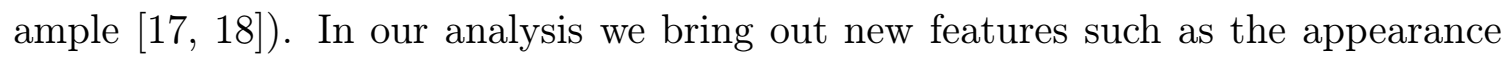
of multiple types of short superconformal multiplets, generic to all orbifolds which break the CFT R-symmetry.

The paper is organized as follows. In section '2in, we describe the singular M-theory geometry conjectured in 191 which yields the $A d S$ dual to the $(1,0)$ theory. Fields in this geometry as well as the operators of the $(1,0)$ theory fall into representations of $O S p(6,2 \mid 2)$. In section $\hat{\beta}_{-1}^{-1}$ we build short multiplets of interest for this supergroup using the oscillator construction of [21. Section $\sin _{-1}^{-1}$ matches $E_{8}$ neutral operators of the $(1,0)$ theory with $\mathrm{K}-\mathrm{K}$ reduced fields from the bulk M-theory geometry, while section ${ }_{-1}^{-5}$ matches operators charged under $E_{8}$ with the $\mathrm{K}-\mathrm{K}$ reduction of fields living at the singular locus of this geometry. Finally in section we summarize by listing relevant and marginal operators of the $(1,0)$ theory, and comment on their physical relevance. We also discuss at some length some general features of orbifold constructions.

\section{Compactification geometry}

The $(1,0)$ theory we are interested in can be thought of as placing a large number, $N$, of M5-branes on top of an "end-of-the-world" 9-branes of the Hořava-Witten compactification of M-theory [200], $R^{1,9} \times S^{1} / \mathbb{Z}_{2}$. The near horizon geometry of $\mathrm{N}$ M5-branes is $A d S_{7} \times S^{4}$, where the $S^{4}$ is described by the equation

$$
\left(X^{6}\right)^{2}+\left(X^{7}\right)^{2}+\left(X^{8}\right)^{2}+\left(X^{9}\right)^{2}+\left(X^{11}\right)^{2}=R^{2} .
$$

$\mathrm{R}$ is a fixed radius whose size grows with $\mathrm{N}$.

\footnotetext{
${ }^{1}$ We label by $\mathrm{N}$ the $(1,0)$ theory which can be resolved to $\mathrm{N} / 2$ free tensor theories in its Coulomb branch
} 
Since the $\mathbb{Z}_{2}$ mentioned above flips the sign of $X^{11}$, it is natural to believe that large $\mathrm{N}(1,0)$ theory is dual to M-theory on $A d S_{7} \times S^{4} / \mathbb{Z}_{2}$ as conjectured in $[\underline{1} \overline{1} \overline{\underline{9}}]$. The $\mathbb{Z}_{2}$ action has a fixed point locus, $A d S_{7} \times S^{3}$, on which propagates the $D=10 \mathcal{N}=1$ SYM $E_{8}$ multiplet derived in the Hořava-Witten compactification. This $E_{8}$ twisted sector was present in the M-theory background before we took the near-horizon limit. It can also be directly motivated, as the gravitational anomaly argument [201] which dictates it's presence at $\mathbb{Z}_{2}$ singularities in flat space still applies in our curved background.

Upon reduction of M-theory down to the $A d S_{7}$, there are two kinds of $\mathrm{K}-\mathrm{K}$ modes. The $\mathrm{K}-\mathrm{K}$ modes of the first kind are given in terms of the $\mathrm{K}-\mathrm{K}$ modes of M-theory on $S^{4}$ which survive the $\mathbb{Z}_{2}$ projection. These modes carry no $E_{8}$ quantum numbers and hence couple only to $E_{8}$-neutral operators on the boundary of $A d S_{7}$. The K-K-modes from the fixed $S^{3}$ have adjoint $E_{8}$ quantum numbers and couple to charged operators on the $A d S_{7}$ boundary, in a manner similar to the one described in [i 1 i . Note that since only adjoint $E_{8}$ fields exist in the bulk, any operator on the boundary in a representation of $E_{8}$ other than the adjoint will have to couple to a multi-particle state. ${ }^{2}$

Two more points need to be made about the modes on this M-theory background. First, the isometry group, $\mathrm{SO}(5)$, of the original $S^{4}$ is broken to $\mathrm{SO}(4)=\mathrm{SU}(2)_{R} \times$ $\mathrm{SU}(2)_{L}$ by the $\mathbb{Z}_{2}$ projection. The gauge group for $A d S_{7}$ is then $\mathrm{SU}(2)_{R} \times \mathrm{SU}(2)_{L} \times E_{8}$, but we pick a basis such that only the first factor is embedded in the superconformal algebra $\operatorname{OSp}(6,2 \mid 2)$. Second, the "twisted sector" $E_{8}$ fields will not shift the large N mass spectrum of the $\mathrm{K}-\mathrm{K}$ bulk modes since they only affect tree-level computations when they are given a non-zero vev. For large $\mathrm{N}$ the size of the $S^{4}$ grows much faster than that of the fixed point $S^{3}$, therefore masses for bulk Kaluza-Klein modes unprotected by supersymmetry will receive at most $1 / \mathrm{N}$ corrections from interactions with the twisted sector fields.

\section{Oscillator construction of $\operatorname{OSp}(6,2 \mid 2)$ short multiplets}

Before going on to calculate the mass spectrum of the low-energy Kaluza-Klein Mtheory modes on the geometry described above, we would like to first take a moment to construct the short multiplets of $\operatorname{OSp}(6,2 \mid 2)$ which will be relevant to our problem. We do this because any $\mathrm{K}-\mathrm{K}$ modes which we can fit into one of these short multiplets will have mass eigenvalues fixed by the $\operatorname{OSp}(6,2 \mid 2)$ group theory, making explicit calculations unnecessary. We follow the methods of Günaydin et al. [2i 1 i] used for constructing multiplets of $\operatorname{OSp}(6,2 \mid 4)$ with just a slight modification to allow for the reduced R-symmetry. Since this method is extensively described in [i21], we will only give a brief review.

\footnotetext{
${ }^{2}$ See [222] for more on the role of multi-particle states.
} 
The basic idea is to start with the maximal compact proper subgroup of the bosonic part of the $\operatorname{OSp}(6,2 \mid 2)$ superalgebra, $\mathrm{SO}(6,2) \times \mathrm{SU}(2)_{R}$. This subgroup is $\mathrm{U}(4)_{B} \times \mathrm{U}(1)_{F}$. We next introduce $p$ pairs of bosonic creation/annihilation operators $a^{i}(r)=\left(a_{i}(r)\right)^{\dagger}$ and $b^{i}(r)=\left(b_{i}(r)\right)^{\dagger}$ transforming in the 4 and $\overline{4}$ representation of $\mathrm{U}(4)_{B}$ (with $\left.r=1, \ldots, p\right)$, as well as a pair of fermionic creation/annihilation operators $\alpha^{\dagger}(r), \alpha(r)$ and $\beta^{\dagger}(r), \beta(r)$ with $\mathrm{U}(1)_{F}$ charges $+1 / 2$ and $-1 / 2$ respectively. We organize these oscillators into column vectors:

$$
\begin{aligned}
& \xi_{A}(r)=\left(\begin{array}{c}
a_{i}(r) \\
\alpha(r)
\end{array}\right), \quad \eta_{A}(r)=\left(\begin{array}{c}
b_{i}(r) \\
\beta(r)
\end{array}\right) \\
& \xi^{A}(r)=\left(\begin{array}{c}
a^{i}(r) \\
\alpha^{\dagger}(r)
\end{array}\right), \quad \eta^{A}(r)=\left(\begin{array}{c}
b^{i}(r) \\
\beta^{\dagger}(r)
\end{array}\right), \quad\left\{\begin{array}{l}
r=1, \ldots, p, \\
i, j, \ldots=1, \ldots, 4
\end{array}\right.
\end{aligned}
$$

( $A=\cdot$ will represent the one fermionic index) whose only non-zero commutation/anti-commutation relations can be represented symbolically as

$$
\left\{\xi_{A}(r), \xi^{B}(s)\right]=\delta_{A}{ }^{B} \delta_{s}^{r}, \quad\left\{\eta_{A}(r), \eta^{B}(s)\right]=\delta_{A}{ }^{B} \delta_{s}^{r}
$$

The Lie superalgebra can now be realized in terms of bilinears in $\xi$ and $\eta$. They are given by

$$
\begin{aligned}
& A_{A B}=\xi_{A} \cdot \eta_{B}-\eta_{A} \cdot \xi_{B}, \\
& A^{A B}=A_{A B}^{\dagger}=\eta^{B} \cdot \xi^{A}-\xi^{B} \cdot \eta^{A}, \\
& M_{B}^{A}=\xi^{A} \cdot \xi_{B}+(-1)^{\operatorname{deg}(A) \operatorname{deg}(B)} \eta_{B} \cdot \eta^{A},
\end{aligned}
$$

where $\operatorname{deg}(A)=0$ for a bosonic index $A$ and $\operatorname{deg}(A)=1$ for a fermionic index $A$. The even subalgebra $\mathrm{SO}(6,2) \times \mathrm{SU}(2)_{R}$ is generated by the elements $\left\{A_{i j}, A^{i j}, M^{i}{ }_{j}\right\}$ and $\left\{A . ., A^{*}, M^{*}.\right\}$.

This oscillator construction of generators naturally implements a Jordan decomposition of $\operatorname{OSp}(6,2 \mid 2)$ with respect to the maximal compact subgroup U(4|1) graded with the $\mathrm{U}(1)$ generator, $Q=\frac{1}{2} M_{A}^{A}$ :

$$
L=L^{-} \oplus L^{0} \oplus L^{+}
$$

The generators $A_{A B}$ and $A^{A B}$ correspond to the $L^{-}$and $L^{+}$spaces, respectively. The generators $M_{B}^{A}$ of $\mathrm{U}(4 \mid 1)$ give the $L^{0}$ space. We use this Jordan decomposition to generate unitary irreducible representations (UIR's) of $\operatorname{OSp}(6,2 \mid 2)$. We start with a lowest weight state, $|\Omega\rangle$, in an irreducible representation of $L^{0}$ and annihilated by all the generators in $L^{-}$. The complete UIR is then generated using successive applications of generators in $L^{+}$on $|\Omega\rangle$. 
To understand the physical interpretation of states in a given UIR, it is convenient to further decompose representations of $\mathrm{U}(4 \mid 1)$ into representations of $\mathrm{U}(4)_{B} \times$ $\mathrm{U}(1)_{F}$. We label the $\mathrm{U}(4 \mid 1)$ representations in terms of super-Young tableaux. ${ }^{3}$ Their decompositions look like:

$$
\measuredangle \rightarrow(\square, 1),(\square, \square),(1, \square)
$$

The group $\mathrm{U}(4)_{B} \simeq \operatorname{Spin}(6) \times \mathrm{U}(1)_{B}$ is the maximal compact subgroup of $\mathrm{SO}(6,2) . \mathrm{U}(1)_{B}$ is generated by the charge $Q_{B}=\frac{1}{2} M_{i}^{i}=\frac{1}{2}\left(N_{B}+4 p\right)$. In the $A d S / C F T$ duality, this charge corresponds to the $A d S$ energy of a supergravity mode and to the dimension of its dual CFT operator. $N_{B}$ is the bosonic number operator, and for a $\mathrm{U}(4)$ representation is just the number of boxes in the corresponding Young tableau. For that given Young tableau, the Spin(6) representation can be recovered by matching the $\mathrm{SU}(4)$ indices with the appropriate $\mathrm{SU}(4)$-invariant tensor. A few examples ${ }^{4}$ are:

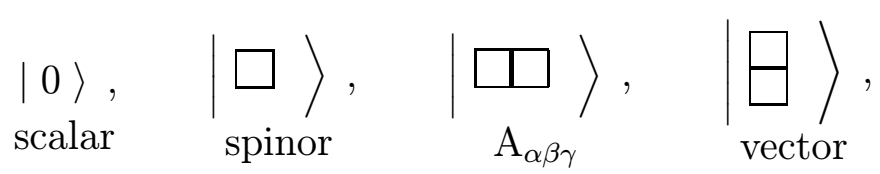

Finally, $\mathrm{U}(1)_{F}$ is generated by $Q_{F}=\frac{1}{2} M^{*}:=\frac{1}{2}\left(N_{F}-p\right)$, which measures $\mathrm{SU}(2)_{R}$ spin.

Now that we have a better understanding of how to interpret states in representations of $\mathrm{U}(4 \mid 1)$, let us quickly describe the action of the $L^{+}$generators. In order to do this, we break up the set $L^{+}$into the subsets:

$$
L^{+}=\left\{A^{A B}\right\} \rightarrow\left\{\begin{array}{l}
B^{+}=\left\{A^{i j}\right\} \\
Q^{+}=\left\{A^{i \cdot}\right\} \\
F^{+}=\left\{A^{\cdot \cdot}\right\}
\end{array}\right.
$$

Given a representation of $\mathrm{U}(4)_{B} \times \mathrm{U}(1)_{F}, B^{+}$gives conformal descendants. In $A d S$ these correspond to higher energy Fourier-like modes. $F^{+}$generates the complete set of spin states in a given $\mathrm{SU}(2)_{R}$ representation. Finally, $Q^{+}$is the set of supersymmetry generators.

To illustrate how the oscillator construction works, we start with the simplest example

$$
|\Omega\rangle=|0\rangle_{p}
$$

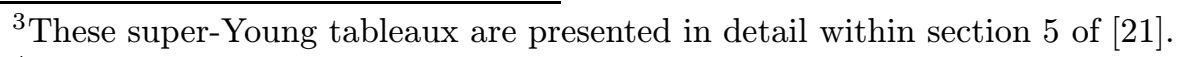

${ }^{4}$ More details can be found at the end of section 금 of [2] $\left.\overline{1}^{\prime}\right]$
} 
the vacuum corresponding to $p$ pairs of oscillators. This state is a scalar with dimension/energy equal to $2 p$ and lowest $\mathrm{SU}(2)_{R}$ spin $-p / 2$. With its $F^{+}$descendants, $|\Omega\rangle$ fills out the $(\mathbf{p}+\mathbf{1})$ of $\mathrm{SU}(2)_{R}$. Acting with $Q$ gives the following supermultiplet of conformal primary states (lowest energy states):

\begin{tabular}{|c|c|c|c|}
\hline $\mathrm{U}(4)$ & State & $\mathrm{SU}(2)_{R}$ & $\Delta$ \\
\hline \hline 1 & Scalar & $(\mathbf{p}+\mathbf{1})$ & $2 p$ \\
\hline$\square$ & Spinor & $\mathbf{p}$ & $\left(2 p+\frac{1}{2}\right)$ \\
\hline 日 & Vector & $(\mathbf{p}-\mathbf{1})$ & $(2 p+1)$ \\
\hline 日 & Spinor & $(\mathbf{p}-\mathbf{2})$ & $\left(2 p+\frac{3}{2}\right)$ \\
\hline 日 & Scalar & $(\mathbf{p}-\mathbf{3})$ & $(2 p+2)$ \\
\hline
\end{tabular}

For $p<4$ we keep only those states with positive $\mathrm{SU}(2)_{R}$ dimension. For readability's sake we always include the $\operatorname{Spin}(6)$ representation labeled in terms of fields in $A d S_{7}$.

The highest spacetime spin in the multiplet ( representation of the superalgebra. In fact, if we act with $Q$ on the superconformal primary, the topmost scalar, its highest weight $\mathrm{SU}(2)_{R}$ component is annihilated. This means that we are dealing with a level one short multiplet in the formalism discussed by [2. $2 \overline{3}$. The superconformal primary state in this type of short multiplet (the topmost scalar) has dimension $\Delta=4 s$ where $s$ is the $\mathrm{SU}(2)_{R}$ spin. We can check this explicitly:

$$
\begin{aligned}
\Delta & =Q_{B}|0\rangle_{p}=2 p \\
& =-4 Q_{F}|0\rangle_{p}=4 s .
\end{aligned}
$$

In order to differentiate from other short multiplets, we call this type of multiplet a chiral multiplet. It will play an important role in our analysis.

There is another short multiplet of $\operatorname{OSp}(6,2 \mid 2)$ that we will find useful, starting with the ground state

$$
\xi^{[A} \xi^{B]}|0\rangle_{p}
$$

This "ground state", with super-Young tableau given in eq. (3..5 of dimension $(2 p+1)$, a spinor of dimension $(2 p+1 / 2)$ and a scalar of dimension $2 p$. Since the scalar has lowest dimension, we will refer to it as the superconformal 
primary. The states in this multiplet are:

\begin{tabular}{|c|c|c|c|}
\hline $\mathrm{U}(4)$ & State & $\mathrm{SU}(2)_{R}$ & $\Delta$ \\
\hline \hline 0 & Scalar & $(\mathbf{p}-\mathbf{1})$ & $2 p$ \\
\hline$\square$ & Spinor & $\mathbf{p}$ & $\left(2 p+\frac{1}{2}\right)$ \\
$(\mathbf{p}-\mathbf{2})$ & \\
\hline$\square$ & 3-Form & $(\mathbf{p}-\mathbf{1})$ & $(2 p+1)$ \\
\hline$\square$ & Vector & $\begin{array}{r}(\mathbf{p}+\mathbf{1}) \\
(\mathbf{1})\end{array}$ & $(2 p+1)$ \\
\hline$\square$ & Gravitino & $\mathbf{p}$ & $\left(2 p+\frac{3}{2}\right)$ \\
\hline$\square$ & $(\mathbf{p}-\mathbf{2})$ & \\
\hline$\square$ & Graviton & $(\mathbf{p}-\mathbf{1})$ & $(2 p+2)$ \\
\hline$\square$ & & $\mathbf{p}$ & \\
\hline$\square$ & Spinor & $\begin{array}{l}(\mathbf{p}-\mathbf{2}) \\
(\mathbf{p}-\mathbf{4})\end{array}$ & $\left(2 p+\frac{3}{2}\right)$ \\
\hline
\end{tabular}

\begin{tabular}{|c|c|c|c|}
\hline $\mathrm{U}(4)$ & State & $\mathrm{SU}(2)_{R}$ & $\Delta$ \\
\hline & 2-Form & $\begin{array}{l}(\mathbf{p}-\mathbf{1}) \\
(\mathbf{p}-\mathbf{3})\end{array}$ & $(2 p+2)$ \\
\hline & Gravitino & $\begin{array}{c}(\mathbf{p}-\mathbf{2}) \\
\mathbf{p}\end{array}$ & $\left(2 p+\frac{5}{2}\right)$ \\
\hline & Scalar & $\begin{array}{l}(\mathbf{p}-\mathbf{1}) \\
(\mathbf{p}-\mathbf{3}) \\
(\mathbf{p}-\mathbf{5})\end{array}$ & $(2 p+2)$ \\
\hline & Spinor & $\begin{array}{l}(p-2) \\
(p-4)\end{array}$ & $\left(2 p+\frac{5}{2}\right)$ \\
\hline 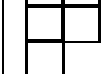 & Vector & $(\mathbf{p}-\mathbf{3})$ & $(2 p+3)$ \\
\hline
\end{tabular}

Again, we only allow states with positive $\mathrm{SU}(2)_{R}$ dimension. Even then, for $p<5$ some of the elements listed above are not in fact conformal primaries, but $F^{+}$and $B^{+}$descendants of other primaries. For example, when $p=2$ the vector in the $\mathbf{1}$ of $\mathrm{SU}(2)_{R}$ is the $F^{+}$descendant of the vector in the 3 . This odd behavior is related to the fact that this multiplet does not develop null states until level 3. The superconformal primary (scalar) for this short multiplet has $\Delta=4 s+4$. We know from [2]-3] that short multiplets of $\operatorname{OSp}(6,2 \mid 2)$ can have scalar superconformal primaries with

$$
\Delta=4 s, 4 s+2,4 s+4,4 s+6,
$$

but only the ones described in this chapter will be relevant for the supergravity spectrum.

\section{Kaluza-Klein reduction of bulk eleven-dimensional super- gravity}

Now that we have established some of the multiplet structure of $\operatorname{OSp}(6,2 \mid 2)$ we can carry out our analysis of the bulk $S^{4}$ Kaluza-Klein modes which survive the $\mathbb{Z}_{2}$ projection. The group theory for this process is simple. These $\mathrm{K}-\mathrm{K}$ modes come from $S^{4}$ harmonics in representations of $\mathrm{SO}(5)$. Decomposing $\mathrm{SO}(5) \rightarrow \mathrm{SU}(2)_{R} \times$ 
$\mathrm{SU}(2)_{L} \times \mathbb{Z}_{2}$, with $\mathbb{Z}_{2}=+$ /- for harmonics which are even/odd under $X^{11} \rightarrow-X^{11}$, we reduce all the 11-dimensional supergravity fields on the even harmonics except for the 3 -form. ${ }^{5}$ This last field is reduced on odd harmonics since it flips sign under parity reversal. For convenience, we will describe the $\mathrm{K}-\mathrm{K}$ spectrum in terms of the dual CFT operators and apply the $\mathbb{Z}_{2}$ projection on the CFT spectrum.

The $S^{4} \mathrm{~K}-\mathrm{K}$ spectrum derived in ref. [24, , 25] can be nicely organized in terms of

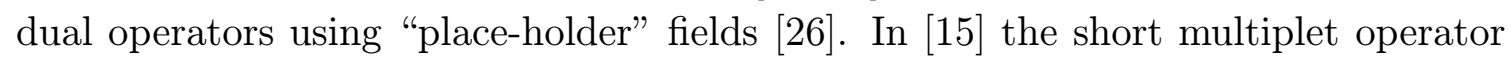
spectrum for the $(2,0)$ theory dual to $A d S_{7} \times S^{4}$ was written in terms of place-holder scalar, spinor and tensor fields

$$
\tilde{\phi}, \quad \tilde{\psi}, \quad \tilde{H},
$$

taken to be in the adjoint of $\mathrm{U}(N)$. They transform in the $\mathbf{5}, \mathbf{4}$, and $\mathbf{1}$ representation ${ }^{6}$ of $\mathrm{SO}(5)$. Starting with a superconformal primary operator

$$
\mathcal{O}_{0,0, p}=\operatorname{Tr} \tilde{\phi}^{p}
$$

(the power $p$ is schematic, the $\tilde{\phi}$ 's are actually in symmetric traceless representation of $\mathrm{SO}(5)$ ) we build a complete supermultiplet of conformal primary operators

$$
\mathcal{O}_{m, n, k}=\operatorname{Tr} \tilde{H}^{m} \tilde{\psi}^{n} \tilde{\phi}^{k}, \quad m+n+k=p .
$$

Note that in the oscillator method used to generate superconformal multiplets of $O S p(6,2 \mid 4)$, these multiplets are built using the vacuum ground state with the same number $p$ of oscillator flavors (see [2픽). The $\mathrm{p}=1$ operators $\operatorname{Tr} \tilde{H}, \operatorname{Tr} \tilde{\psi}$, and $\operatorname{Tr} \tilde{\phi}$ are the only operators in the Abelian part of $\mathrm{U}(N)$ and correspond to the doubleton. They decouple from the theory. The $p=2$ supermultiplet (also referred to as the massless multiplet) is very important, as it contains the R-symmetry currents, the super-currents and the stress-energy tensor, as well as relevant scalar operators.

To look at the operators dual to the $\mathrm{K}-\mathrm{K}$ modes of $S^{4} / \mathbb{Z}_{2}$ we use a simple extension of the methods above. We split the place-holder fields into an even group and an odd group. The even group contains scalars and spinors

$$
\begin{aligned}
& \phi \quad \text { in the }(\mathbf{2}, \mathbf{2}), \\
& \psi \quad \text { in the }(\mathbf{1}, \mathbf{2}),
\end{aligned}
$$

where $\mathrm{SU}(2)_{R} \times \mathrm{SU}(2)_{L}$ is now the global symmetry. These place-holders transform in the anti-symmetric (AS) of $\operatorname{USp}(N)$ ( $N$ must be even) and loosely correspond to fluctuations inside the end-of-the-world 9-brane. Since we have broken $\mathrm{U}(N)$ to $U S p(N)$, traces of place-holder fields include the symplectic matrix $\mathbf{J}$. This means any number of these even place-holder fields can appear in a trace $(\operatorname{Tr}[\mathbf{J} \cdot \mathbf{A S}] \neq 0)$.

\footnotetext{
${ }^{5}$ Here we refer to forms in index free notation

${ }^{6}$ We denote with a fields in $\mathrm{SO}(5)$ R-symmetry representations
} 
The odd group contains a scalar, spinors, and a self-dual 2-form

$$
\begin{aligned}
& \rho \quad \text { in the }(\mathbf{1}, \mathbf{1}), \\
& \chi \quad \text { in the }(\mathbf{2}, \mathbf{1}), \text { and } \\
& H \quad \text { in the }(\mathbf{1}, \mathbf{1}) .
\end{aligned}
$$

These place-holders transform in the adjoint of $\operatorname{USp}(N)$, so only operators represented by traces with an even number of these survive the $\mathbb{Z}_{2}$ projection due to the commutation relation of adjoint $\operatorname{USp}(N)$ matrices with $\mathbf{J}$. For a given $p$, the superconformal primaries $\mathcal{O}_{0,0, p}$ of the $\mathcal{N}=2$ algebra break up into separate superconformal primaries of the $\mathcal{N}=1$ algebra. The ones which survive the $\mathbb{Z}_{2}$ projection can be schematically written as

$$
\operatorname{Tr} \phi^{p}, \operatorname{Tr} \phi^{p-2} \rho^{2}, \operatorname{Tr} \phi^{p-4} \rho^{4}, \ldots
$$

The first of these primaries will transform in the $(\mathbf{p}+\mathbf{1}, \mathbf{p}+\mathbf{1})$ of $\mathrm{SU}(2)_{R} \times \mathrm{SU}(2)_{L}$ the next in the $(\mathbf{p}-\mathbf{1}, \mathbf{p}-\mathbf{1})$, and so on. They all inherit the dimension of $\mathcal{O}_{0,0, p}$, so will have $\Delta=2 p$. Relating their R-symmetry spin, $s$, to this dimension we get the relations:

$$
\Delta=4 s, \quad \Delta=4 s+4, \quad \Delta=4 s+8, \ldots
$$

For each $p$, the first operator in this series is a superconformal primary for a chiral short multiplet with content as in ( $\left(\overline{3} . \bar{n}_{1}^{\prime}\right)$. The second is also an $\mathcal{N}=1$ superconformal primary for a short multiplet, but now with the primary fields listed in ( rest of the operators in ( $\left.\bar{A}_{-} \bar{z}_{1}\right)$ are superconformal primaries for long multiplets of $\operatorname{OSp}(6,2 \mid 2)$. This can be seen both from their dimension, scalars with $\Delta>4 s+6$ are superconformal primaries for long multiplets only, and from the number of states in the multiplet. Their dimensions are valid only in the large $\mathrm{N}$ limit, since they can receive corrections of order $1 / \mathrm{N}$ when tree-level supergravity computations are no longer protected by supersymmetry.

For $p=1$, only one superconformal operator can be written down, $\operatorname{Tr} \phi$. The conformal primaries in this multiplet are this scalar in the $\mathbf{( 2 , 2 )}$ of $\mathrm{SU}(2)_{R} \times \mathrm{SU}(2)_{L}$ and a spinor in the $(\mathbf{1 , 2})$. The anti-symmetric tensor of $\operatorname{USp}(N)$ is reducible, its symplectic trace is a singlet and won't mix with other operators. This matches with our understanding of the $p=1$ operators as dual to the "pure gauge" (i.e. no physical degrees of freedom) $A d S$ fields in the doubleton representation of $\operatorname{OSp}(6,2 \mid 2)$. They correspond to the center of mass motion of the M5-branes parallel to the 9-branes and decouple. ${ }^{7}$

We would like to point out that a clear distinction should be drawn between place-holder fields and doubleton degrees of freedom. In the $\mathcal{N}=2$ formalism, the

\footnotetext{
${ }^{7}$ This decoupling of the center of mass degrees of freedom follows from coordinate invariance inside the 9-brane.
} 
doubleton and the place-holder multiplet carry the same quantum numbers, so an identification might be drawn. For $\mathcal{N}=1$ only the even multiplet of place-holders yields a doubleton, the odd place-holders are necessary for computing the correct spectrum but can't appear alone in a trace. Thus, the pure-gauge doubleton degrees of freedom of the $A d S$ background should really only be interpreted as decoupled center of mass degrees of freedom for the CFT and bear no direct connection to the place-holder fields.

The odd place-holders first play a role for the $p=2$ operators. The $\mathcal{N}=2$ super-primary operator $\operatorname{Tr} \tilde{\phi} \tilde{\phi}$ splits into $\operatorname{Tr} \phi \phi, \operatorname{Tr} \phi \rho$, and $\operatorname{Tr} \rho \rho$. Only the first and third of these survive the $\mathbb{Z}_{2}$ projection. We list the results in the following table:

\begin{tabular}{|l|c|l|l|ll|}
\hline Type & $\Delta$ & $\mathrm{SO}(5) \rightarrow \mathrm{SU}(2)_{R} \times \mathrm{SU}(2)_{L} \times \mathbb{Z}_{2}$ & \multicolumn{2}{|c|}{ Place-Holders } \\
\hline \hline Scalar & 4 & $\mathbf{1 4} \rightarrow(\mathbf{3}, \mathbf{3})_{+}+(\mathbf{1}, \mathbf{1})_{+}+\mathbb{Z}_{2}$-odd & $\operatorname{Tr} \tilde{\phi} \tilde{\phi} \rightarrow$ & $\operatorname{Tr} \phi \phi+\operatorname{Tr} \rho \rho+\mathbb{Z}_{2}$-odd \\
\hline Spinor & $4 \frac{1}{2}$ & $\mathbf{1 6} \rightarrow(\mathbf{2}, \mathbf{3})_{+}+(\mathbf{2}, \mathbf{1})_{+}+\mathbb{Z}_{2}-$ odd & $\operatorname{Tr} \tilde{\phi} \tilde{\psi} \rightarrow$ & $\operatorname{Tr} \phi \psi+\operatorname{Tr} \rho \chi+\mathbb{Z}_{2}$-odd \\
\hline Vector & 5 & $\mathbf{1 0} \rightarrow(\mathbf{1}, \mathbf{3})_{+}+(\mathbf{3}, \mathbf{1})_{+}+\mathbb{Z}_{2}$-odd & $\operatorname{Tr} \tilde{\psi} \tilde{\psi} \rightarrow$ & $\operatorname{Tr} \psi \psi+\operatorname{Tr} \chi \chi+\mathbb{Z}_{2}$-odd \\
\hline 3-Form & 5 & $\mathbf{5} \rightarrow$ & $(\mathbf{1}, \mathbf{1})_{+}+\mathbb{Z}_{2}$-odd & $\operatorname{Tr} \tilde{\phi} \tilde{H} \rightarrow$ & $\operatorname{Tr} \rho H+\mathbb{Z}_{2}$-odd \\
\hline Gravitino & $5 \frac{1}{2}$ & $\mathbf{4} \rightarrow$ & $(\mathbf{2}, \mathbf{1})_{+}+\mathbb{Z}_{2}$-odd & $\operatorname{Tr} \tilde{\psi} \tilde{H} \rightarrow$ & $\operatorname{Tr} \chi H+\mathbb{Z}_{2}$-odd \\
\hline Graviton & 6 & $\mathbf{1} \rightarrow$ & $(\mathbf{1}, \mathbf{1})_{+}+\mathbb{Z}_{2}$-odd & $\operatorname{Tr} \tilde{H} \tilde{H} \rightarrow$ & $\operatorname{Tr} H H+\mathbb{Z}_{2}$-odd \\
\hline
\end{tabular}

From this table, we can see that we get two massless multiplets for $A d S_{7} \times S^{4} / \mathbb{Z}_{2}$. The first is a chiral multiplet and contains the $\mathrm{SU}(2)_{L}$ gauge field. The second multiplet contains the $\mathrm{SU}(2)_{R}$ gauge field, the gravitino, and the graviton. These fields couple to the R-symmetry current, the super-current and the stress-energy tensor of the boundary CFT respectively. The presence of the R-symmetry current and the stress-energy tensor in the same multiplet fixes the relation between R-spin, $s$, and the dimension, $\Delta$, of the short multiplets superconformal primaries. ${ }^{8}$

The rest of the $(1,0)$ spectrum dual to the $A d S_{7} \times S^{4} / \mathbb{Z}_{2}$ bulk fields can be easily obtained using the place-holder fields described above. The group theory for projecting down $\mathcal{N}=2$ multiplets is straightforward and this part of our analysis requires no new understanding of the $\mathrm{K}-\mathrm{K}$ reduction of 11-dimensional supergravity. Because the complete Kaluza-Klein spectrum on $S^{4}$ has already been worked out in $[\overline{2} \overline{4},, 2 \overline{2} 5]$, we only need to identify those bulk states which survive the $\mathbb{Z}_{2}$ projection. It's important to note that since we do not have a lagrangean UV description for the $(1,0)$ theory, the place-holder formalism is only a labeling system convenient for doing group theory.

\footnotetext{
${ }^{8}$ The fact that the various short multiplets relate $\Delta$ and $s$ differently is due to the appearance of null-states at different levels.
} 


\section{Kaluza-Klein reduction of $E_{8}$ twisted sector modes}

To get the mass spectrum of the Kaluza-Klein reduction of the $E_{8}$ twisted sector modes, one might expect to have to calculate the eigenvalues of the Laplace operator for the $E_{8} \mathcal{N}=1 D=10$ vector multiplet reduced on a sphere. Luckily, group theory comes to our rescue again, in a fashion quite similar to that in the analysis of [i] $\left.{ }_{1}^{1} \overline{8}\right]$. Upon reduction to $A d S_{7}$ the $D=10$ vector multiplet can give only scalars, spinors, and vectors. This implies that its $\mathrm{K}-\mathrm{K}$ modes must fit into chiral short multiplets, since all other multiplets include a larger set of bosonic fields.

Looking at just the bosonic modes, we derive the complete Kaluza-Klein spectrum. Our only initial bosonic field is the $D=10$ vector field. It can be reduced on the fixed $S^{3}$ using a scalar harmonic in the $(\mathbf{k}, \mathbf{k})$ of $\mathrm{SU}(2)_{R} \times \mathrm{SU}(2)_{L}$ or using a vector harmonic in the $(\mathbf{k}+\mathbf{2}, \mathbf{k})$ or $(\mathbf{k}, \mathbf{k}+\mathbf{2})$. These harmonics give a bosonic spectrum of the form

$$
\begin{aligned}
& \text { Scalars } \rightarrow(\mathbf{3}, \mathbf{1}),(\mathbf{4}, \mathbf{2}),(\mathbf{5}, \mathbf{3}),(\mathbf{6}, \mathbf{4}), \ldots \\
& \text { Vectors } \rightarrow(\mathbf{1}, \mathbf{1}),(\mathbf{2}, \mathbf{2}),(\mathbf{3}, \mathbf{3}),(\mathbf{4}, \mathbf{4}), \ldots \\
& \text { Scalars } \rightarrow \quad(\mathbf{1}, \mathbf{3}),(\mathbf{2}, \mathbf{4}), \ldots
\end{aligned}
$$

The columns in this series fit easily into chiral multiplets with superconformal primary scalars in the $(\mathbf{k}+\mathbf{2}, \mathbf{k})$. The $A d S$ energy/dimensions of all these states can be directly read off from (3. $(\mathbf{k}+\mathbf{2}, \mathbf{k})$ and $(\mathbf{k}, \mathbf{k}+\mathbf{2})$ have different $A d S$ energies due to a subtlety involving the eigenvalues of the $E_{8}$ covariant derivative on $S^{3}$.

The first multiplet in the series contains the massless vector which carries the $E_{8}$ gauge symmetry and couples to the dual $E_{8}$ current algebra on the $A d S_{7}$ boundary CFT. Since this vector comes from a straight reduction on $S^{3}$, its coupling is proportional to

$$
1 / \sqrt{\operatorname{Vol}\left(S^{3}\right)} \simeq 1 / \sqrt{N}
$$

\section{Discussion}

We conclude by giving a summary of chiral gauge invariant relevant and marginal operators for the $(1,0)$ theory. We make some general remarks on orbifolds in the $A d S / C F T$ context, and offer some perspective on the Coulomb/Higgs branch transition.

We denote operators by their $\mathrm{SU}(2)_{R} \times \mathrm{SU}(2)_{L} \times E_{8}$ quantum numbers. The relevant and marginal scalar operators are:

$$
\begin{aligned}
& (\mathbf{3}, \mathbf{3}, \mathbf{1}),(\mathbf{1}, \mathbf{1}, \mathbf{1}),(\mathbf{3}, \mathbf{1}, \mathbf{2 4 8}), \quad \text { with } \Delta=4(\text { relevant }) \\
& (\mathbf{4}, \mathbf{4}, \mathbf{1}),(\mathbf{2}, \mathbf{2}, \mathbf{1}),(\mathbf{4}, \mathbf{2}, \mathbf{2 4 8}), \quad \text { with } \Delta=6 \text { (marginal }) .
\end{aligned}
$$


All these scalar operators break supersymmetry since they are superconformal primary operators and thus are not annihilated by any supersymmetry transformations. Also, from the analysis of [27] for the relevant scalars of the $(2,0)$ theory, we don't expect the $(\mathbf{3}, \mathbf{3}, \mathbf{1})$ and $(\mathbf{1}, \mathbf{1}, \mathbf{1})$ operators to lead to any new stable superconformal fixed points. On the other hand, the $(\mathbf{3 , 1 , 2 4 8 )}$ scalar operator will very likely be implicated in breaking the $(1,0)$ theory to its Higgs branch, as any $E_{8}$ instanton bundle on the fixed $S^{3}$ will excite the corresponding Kaluza-Klein mode. This analysis requires a solution of the coupled twisted sector and bulk modes of $A d S_{7} \times S^{4} / \mathbb{Z}_{2}$ and is beyond the scope of our discussion.

The rest of the relevant and marginal spectrum behaves as expected. The vector spectrum yields all the expected dimension five global symmetry currents for $\mathrm{SU}(2)_{R} \times \mathrm{SU}(2)_{L} \times E_{8}$. We also find the super-current and the stress-energy tensor and a relevant 3 -form current. An important check on our analysis is that only the $\mathrm{SU}(2)_{R}$ current appears in the same multiplet as the stress-energy tensor. Since this is the only R-symmetry current, anything else would violate the supersymmetry algebra. Continuing with this logic, the $\mathrm{SU}(2)_{L}$ and $E_{8}$ symmetry currents need to appear in a different kind of short multiplet (one without the stress-energy tensor). Clearly, differentiation in the types of short multiplets must appear in any situation involving CFT's with global symmetries that are not R-symmetries.

One way to understand the appearance of new short multiplets for orbifolds of $A d S / C F T$ configurations is by looking more closely at the role of R-symmetry in the shortening process. For example, in the $(2,0)$ spectrum we start with a superconformal primary operator in the symmetric-traceless of the $\mathrm{SO}(5) \mathrm{R}$-symmetry. Only the highest weight state in this representation is annihilated by the supersymmetry transformation, but this is enough to shorten the superconformal multiplet. When we orbifold to get the $(1,0)$ theory this single $\mathrm{SO}(5)$ representation breaks into multiple surviving $\mathrm{SU}(2)_{R} \times \mathrm{SU}(2)_{L}$ representations. If we rank these representations in order of decreasing $\mathrm{SU}(2)_{L}$ dimension, the first contains the highest weight state of $\mathrm{SO}(5)$ and shortens at level 1 . The next (surviving) representation has a state which annihilates at level 3, and so is the primary for a new kind of short multiplet. Since we can't apply the supersymmetry generators an infinite number of times (they are fermionic), a large enough initial $\mathrm{SO}(5)$ representation for the superconformal primary will spawn superconformal primaries for multiplet that never shorten, i.e. long multiplets.

The appearance of these long multiplets in the Kaluza-Klein reduced supergravity single particle spectrum of orbifolds is in itself an interesting occurrence. At tree level the states in the long multiplets inherit their masses from their $(2,0)$ theory progenitors. For large N, these will then be the correct masses, but not necessarily for small N. By contrast, in the standard story for $A d S / C F T$ duality, with maximally symmetric spaces, single particle supergravity states are mapped strictly to operators in short multiplets, i.e. with fixed dimensions. In the large $\mathrm{N}$ limit where 
supergravity is valid only these operators keep finite dimensions, all other operators have anomalous dimensions that grow with $\mathrm{N}$. In orbifolds, the new long multiplets provide us with an extra set of single particle operators, whose naive dimension becomes increasingly correct for large N. This is the kind of behavior usually associated with multi-trace operators dual to multiple particle supergravity states, where the interaction energy correction to the naive mass estimate vanishes in the large $\mathrm{N}$ limit (the coupling goes to zero).

The appearance of exotic multiplets in the single particle spectrum of orbifolds can also be related to the introduction of separate sets of place-holder fields for the dual CFT. In section 离, we separated place-holders into an even and an odd set. The shortest multiplet (denoted chiral) had all even place-holders, the next shortest had two odd place-holders, and so on. We expect this to generalize to other orbifolds as follows. The place-holder fields of the covering space theory will be grouped by their transformation properties under the defining orbifold quotient operation. Preserved operators will correspond to invariant combinations of these place-holder fields. If more than one type of invariant combination exists (e.g., even fields, or odd-odd fields), different types of multiplets should ensue. Note that only placeholder fields which are singlets under the orbifold operation yield doubleton fields in $A d S$. Therefore, the naive connection between place-holders and doubletons seen in maximally symmetric spaces breaks down.

Returning to specifics of the $(1,0)$ theory, we would like to take a look at the flat directions discussed in the introduction. One problem with the notion of moduli space inherent in that discussion is that it is usually parameterized in terms of expectation values of fields. In the conformal field theory language we can only perturb our vacuum by adding operators to the theory. The coefficients for these additional operators then act as parameters. Motion along one of the flat directions of moduli space typically corresponds to adding marginal operators which are not gauge invariant, and as such were not analyzed in our discussion.

Despite this problem it is still possible to look at the moduli space of the $(1,0)$ theory in the near vicinity of the interacting superconformal fixed point. The idea is to move a short distance along moduli space before taking the near-horizon limit. For example, if we separate the stack of $\mathrm{N}$ M5-branes into two stacks of N/2 M5-branes, we can get a multi-center $A d S$ space describing motion along the Coulomb branch. Due to the nature of scaling limit, motion away from the 9branes will always be limited to be less than $l_{P}$, so recovery of the $(2,0)$ theory of N/2 M5-branes is an infinite distance away in moduli space. Similarly, one can hope to analyze the Higgs branch by "blowing-up" $E_{8}$ instantons on the fixed point $S^{3}$. This involves solving coupled twisted sector/bulk equations on $A d S_{7} \times S^{4} / \mathbb{Z}_{2}$, and presents an interesting problem in itself. Once again, the scaling limit which yields the $A d S / C F T$ correspondence only allows us to travel a short distance along this flat direction. 


\section{Acknowledgments}

We would like to thank P. Hořava, C.V. Johnson, S. Kachru, A. Lawrence, J. Maldacena, J. Minahan, D. Minic, S. Minwalla, and J. Schwarz for discussions and helpful comments. This work was supported in part by the U.S. Dept. of Energy under Grant no. DE-FG03-92-ER 40701.

\section{References}

[1] J. Maldacena, The large $N$ limit of superconformal field theories and supergravity,

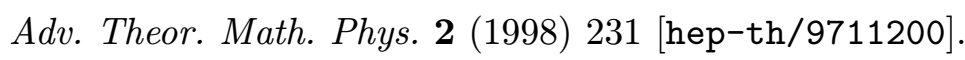

[2] S.S. Gubser, I.R. Klebanov and A.M. Polyakov, Gauge theory correlators from non-

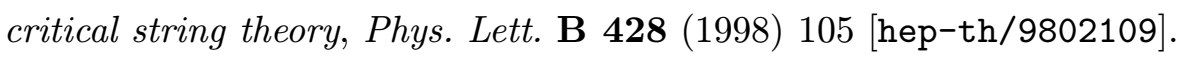

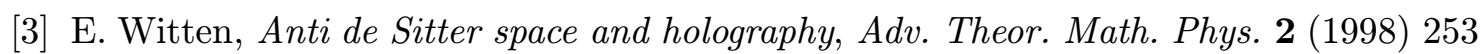
[hep-th/9802150in.

[4] O. Ganor and A. Hanany, Small $E_{8}$ Instantons and Tensionless Noncritical Strings, [Nucl. Phys. B 474 (1996) 122 [hep-th/9602120].

[5] N. Seiberg and E. Witten, Comments on String Dynamics in Six Dimensions, iNuche: (

[6] S. Kachru and E. Siverstein, Chirality-Changing Phase Transitions in $4 D$ String Vacua, iNucl. Phys. B 504 (1997) 272i [nep-th/9704185].

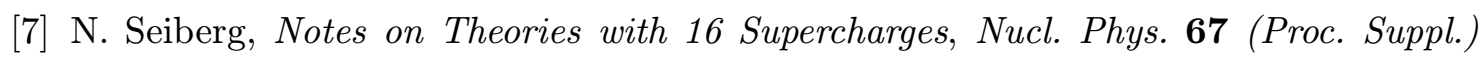
(1)

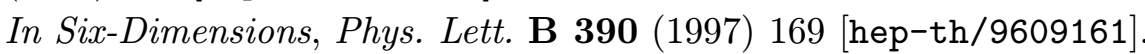

[8] O. Aharony, M. Berkooz, S. Kachru and E. Silverstein, Matrix Descriptions of $(1,0)$

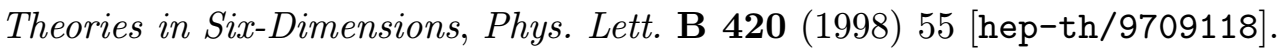

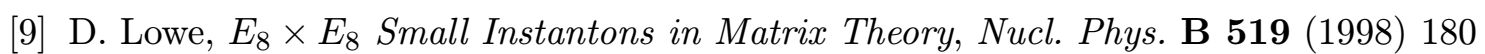

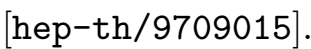

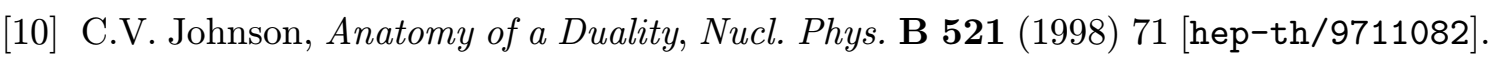

[11] S. Ferrara, A. Kehagias, H. Partouche and A. Zaffaroni, Membranes and five-branes

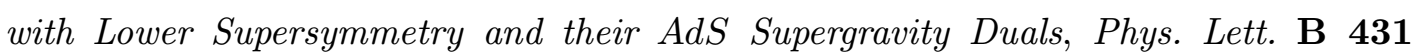
ing

[12] C. Ahn, K. Oh and R. Tatar, Orbifolds of $A d S_{7} \times S^{4}$ and Six Dimensional $(0,1) S C F T$,

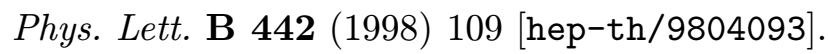

[13] O. Aharony, Y. Oz, Z. Yin, $M$ Theory on $A d S_{p} \times S^{11-p}$ and Superconformal Field

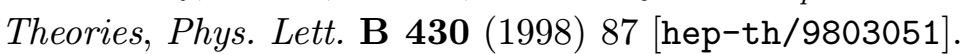


[14] S. Minwalla, Particles on $A d S_{4 / 7}$ and Primary Operators on $M_{2 / 5}$ brane Worldvol-

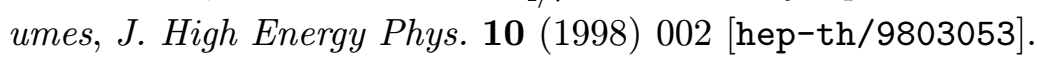

[15] R.G. Leigh and M. Rozali, The Large $N$ Limit of the $(2,0)$ Superconformal Field

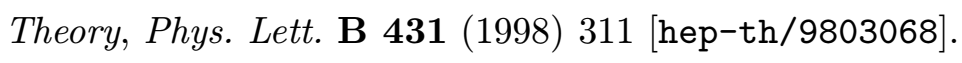

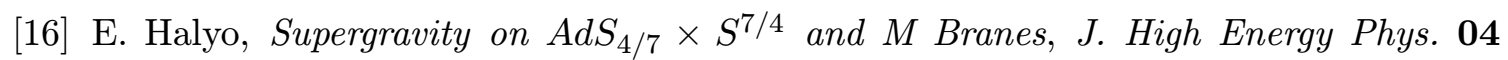
:

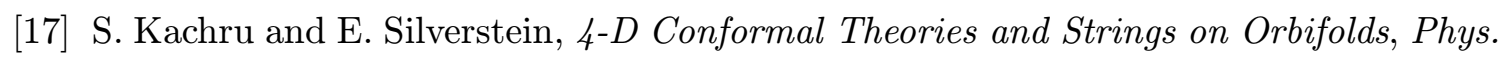

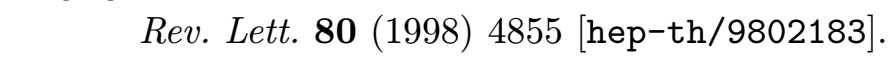

[18] O. Aharony, A. Fayyazuddin and J. Maldacena, The Large $N$ Limit of $\mathcal{N}=2,1$ Field Theories from Threebranes in F-theory, 'J High Energy Phys 07 (1999) 013i [hep-th/9806159i.

[19] M. Berkooz, A supergravity dual of a $(1,0)$ field theory in six dimensions, Phys. Letet. :

[20] P. Hořava and E. Witten, Heterotic and Type I string dynamics from eleven dimen-

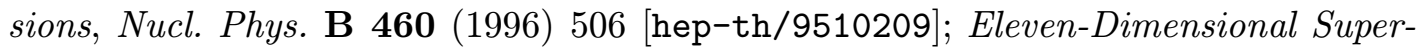

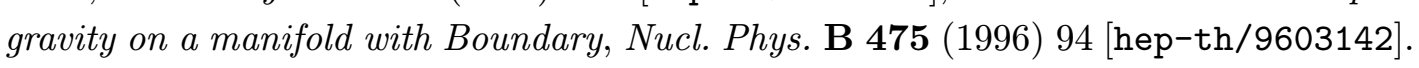

[21] M. Günaydin, P. van Nieuwenhuizen and N. P. Warner, General Construction of the Unitary Representations of Anti-de sitter Superalgebras and the Spectrum of the $S^{4}$ Compactification of 11-Dimensional Supergravity, Nucl. Phys. B. 255.1985) 63.

[22] J. de Boer, Six-Dimensional Supergravity on $S^{3} \times A d S_{3}$ and $2 d$ Conformal Field Theory, hep-th/9806104.

[23] S. Minwalla, Restrictions Imposed by Superconformal Invariance on Quantum Field

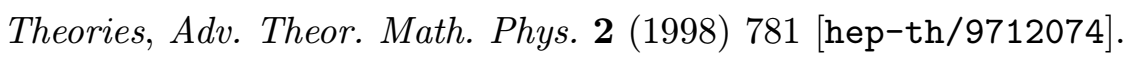

[24] K. Pilch, P.K. Townsend and P. van Nieuwenhuizen, Compactification of $d=11$ supergravity on $S^{4}$ (or $11=7+4$, too), Nucl. Phys. B 242 (1984) 377 .

[25] P. van Nieuwenhuizen, The complete mass spectrum of $d=11$ supergravity compactified on $S_{4}$ and a general mass formula for arbitrary cosets $M_{4}$, 'Class. and Q Quant. Grav.' - .

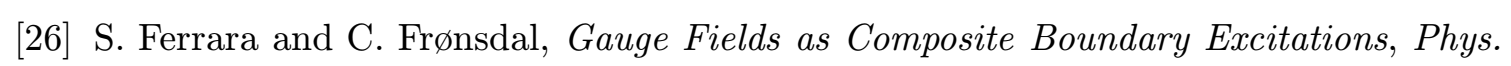

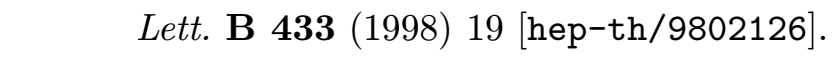

[27] J. Distler and F. Zamora, Nonsupersymmetric Conformal Field Theories from Stable Anti-De Sitter Spaces, 'Adv. Theor. Math. Phys. 2 (1998) 1405: [hep-th/9810206']. 